\title{
Stochastic Generalized Active Space Self-Consistent Field: Theory and Application
}

\author{
Oskar Weser \\ Kai Guther \\ Khaldoon Ghanem \\ Giovanni Li Manni
}

\section{Computational Details}

\subsection{Benzene stack}

Generally contracted atomic natural orbitals (ANO-RCC) of split-valence, double- $\zeta$ plus polarization quality were used (ANO-RCC-VDZP)[1, 2], resulting in a total of 570 basis functions. Scalar relativistic effects were included with the Douglas-Kroll-Hess integral correction. Evaluation of electron repulsion integrals has been accelerated by using the resolution-of-identity Cholesky decomposition with a threshold of $1 \cdot 10^{-3} E_{\mathrm{h}}$. [3-7] For the SCF procedure, a convergence threshold of $1 \cdot 10^{-4} E_{\mathrm{h}}$ over the energy was utilized. The Stochastic-GAS calculations were performed using our new GAS-PCHB algorithm, while the full CAS calculations were performed using the conventional i-FCIQMC algorithm with PCHB excitation generator. A maximum walker number of $2 \cdot 10^{8}$ has been utilized. The semi-stochastic space consisted of $1 \cdot 10^{4}$ configurations. The adaptive shift method was employed with a offset value $\Delta=S / 2$, where $S$ is the shift value. RDMs were sampled using the non-variational initiator approximation expression:

$$
\bar{d}_{P Q R S}=\sum_{D_{\text {init }} \in I} \frac{\left\langle D_{\text {init }}\left|a_{P}^{\dagger} a_{R}^{\dagger} a_{S} a_{Q}\right| \Psi_{\text {total }}\right\rangle}{\left\langle D_{\text {init }} \mid \Psi_{\text {total }}\right\rangle}
$$

as already reported in reference [8]. The energies reported in this manuscript were calculated from these RDMs.

Coordinates The cartesian coordinates for a distance of $4.5 \AA$ can be found in benzene_4.5_Ang.xyz.

Molcas input The OpenMolcas input to prepare the electronic integrals and to perform the orbital SCF optimization is give in benzene_4.5_Ang.inp. 
$\mathrm{NECl}$ input The NECI input for solving the CI problem with FCIQMC is given in benzene_4.5_Ang.FciInp. To vary the interspace excitations the arguments to the GAS-SPEC keyword have to be adjusted.

Starting orbitals The starting orbitals are supplied in the OpenMolcas RasOrb fileformat. The file is called benzene_stack. InpOrb. These orbitals come from five times repeating the MO coefficient matrix of a $(6,6) \mathrm{CASSCF}$ calculation on a single benzene. The orbitals are not orthonormal. The given Molcas input orthonormalizes the starting orbitals depending on the distance of fragments and the resulting AO-overlap.

\section{2 $\mathrm{MRCl}$ on Fe-porphyrin}

All calculations were performed on the same $\mathrm{Fe}^{(\mathrm{II})}$-porphyrin model complex as in our earlier work. $[8,9]$ The pyrrole $\beta$-carbon atoms were removed and remaining bonds were capped with $\mathrm{H}$-atoms. The $\mathrm{Fe}-\mathrm{N}$ bond length was kept at $1.989 \AA$ which is closer to the triplet's equilibrium geometry. The molecule was placed into the $x y$ plane with ligand $\mathrm{N}$-atoms on the $x$ and $y$ axes. The point group of the planar molecule is $D_{4 h}$, however, calculations were carried out using the $D_{2 h}$ Abelian point group, the largest our programs can handle.

Basis set and integral specifications have been chosen as in our earlier work [9]. Generally contracted atomic natural orbitals $(\mathrm{ANO}-\mathrm{RCC})$ of split-valence triple- $\zeta$ plus polarization quality, were utilized (ANO-RCC-VTZP) [1, 2], resulting in a total of 707 basis functions. Scalar relativistic effects were included with the Douglas-Kroll-Hess integral correction. Evaluation of electron repulsion integrals has been accelerated by using the resolution-ofidentity Cholesky decomposition with a threshold of $1 \cdot 10^{-3} E_{\mathrm{h}} \cdot[3-7]$

The FCIQMC RASCI-optimization was performed using our new GAS-PCHB excitation generator with initiator approximation and a maximum walker number of $1 \cdot 10^{9}$. The semi-stochastic space consisted of $1 \cdot 10^{7}$ configurations. The adaptive shift method was applied with an offset of half the correlation energy. The projected energy is reported for the energy results.

Due to the large scale of this calculation we also want to report the computational cost of the FCIQMC dynamics with $1 \cdot 10^{9}$ walkers. They were performed on the Max-Planck Computing and Data Facility (MPCDF) HPC system Cobra using 256 nodes, each having two Intel Xeon Gold 6148 (20 cores, 2.4 GHz) processors and $192 \mathrm{~GB}$ DDR4 RAM, with the nodes connected through Intel OmniPath interconnect. The calculations used 38 processes and $170 \mathrm{~GB}$ of memory per node and ran for $5 \cdot 23 \mathrm{~h}$ resulting in a total of $1.1 \cdot 10^{6}$ core hours. Since NECI is near-linearly parallelized[10], the dynamics with fewer walkers were performed on in-house clusters.

Coordinates The cartesian coordinates can be found in FePor.xyz.

Molcas input The OpenMolcas inputs to prepare the electronic integrals and to perform the orbital SCF optimizations for the two spin states can be found in quint.inp and tripl.inp. 
$\mathrm{NECl}$ input The NECI inputs for solving the CI problem with FCIQMC for the two spin states can be found in quint.FciInp and tripl.FciInp.

Starting orbitals The $(32,34)$ CASSCF converged starting orbitals are supplied in the OpenMolcas RasOrb fileformat. The files are called quint. InpOrb and tripl. InpOrb for the two respective spin states.

\subsection{Spin exchange in $\mathrm{Fe}_{4} \mathrm{~S}_{4}$}

$\mathrm{N}_{4}$ The basis set consisted of generally contracted minimal atomic natural orbitals (ANO-RCC-MB), resulting in a total of 20 basis functions.[1, 2] The calculations were performed with conventional CI in a spin pure basis using OpenMolcas and with FCIQMC in an SD basis using NECI.[10, 11]

The FCIQMC CI-optimization was performed using our new GAS-PCHB and the existing FCI PCHB excitation generator with initiator approximation and a maximum walker number of $1 \cdot 10^{5}$. The semi-stochastic space consisted of $1 \cdot 10^{5}$ configurations. The projected energy is reported for the energy results.

$\mathrm{Fe}_{4} \mathrm{~S}_{4}$ The cluster is depicted in Figure 1.

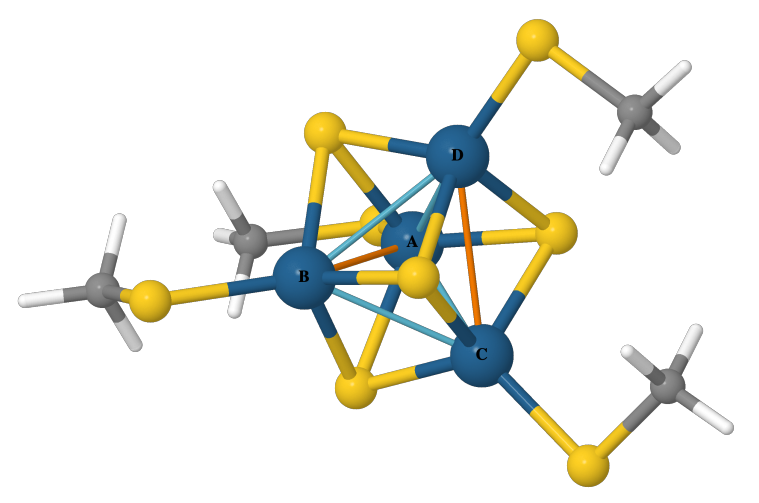

Figure 1: Structure of the $\mathrm{Fe}_{4} \mathrm{~S}_{4}$ cluster.

The basis set consisted of generally contracted minimal atomic natural orbitals on all atoms except iron, where split-valence, double- $\zeta$ plus polarization quality functions were used (ANO-RCC-MB, Fe.ANO-RCC-VDZP)[1, 2], resulting in a total of 212 basis functions.

The FCIQMC CI-optimization was performed using our new GAS-PCHB and the existing FCI PCHB excitation generator with initiator approximation and a maximum walker number of $5 \cdot 10^{7}$. The semi-stochastic space consisted of $1 \cdot 10^{4}$ configurations. The projected energy is reported for the energy results.

Coordinates The coordinates are saved in Fe4S4.xyz. 
Molcas input The OpenMolcas input to prepare the electronic integrals and to perform the orbital SCF optimization for the $s=0$ state can be found in Fe4S4.inp. The input has to be adjusted for the other spin states. The spin argument changes the spin and the FileOrb argument changes the file for the input orbitals.

$\mathrm{NECl}$ input The NECI input for solving the CI problem with FCIQMC is given in Fe4S4 .FciInp. To vary the interspace excitations the arguments to the GAS-SPEC keyword have to be adjusted.

Starting orbitals The CASSCF optimized starting orbitals are supplied in the OpenMolcas Ras0rb fileformat. The files are called Fe4S4_S_\$ $\{$ s\}. Inp0rb where $\$\{\mathrm{~s}\}$ goes from 0 to 10 and denotes the spin state. 


\section{Results}

Table 1: Results for the stack of benzene.

\begin{tabular}{|c|c|c|c|c|}
\hline$d / \AA$ & $n_{\text {exc }}$ & $E / E_{\mathrm{h}}$ & $\mathrm{SCF}$ & constraints \\
\hline 3.0 & 0 & -1154.504927 & $\mathrm{~T}$ & cumulative \\
\hline 3.0 & 1 & -1154.513659 & $\mathrm{~T}$ & cumulative \\
\hline 3.0 & 1 & -1154.513630 & $\mathrm{~T}$ & local \\
\hline 3.0 & 2 & -1154.513777 & $\mathrm{~T}$ & cumulative \\
\hline 3.0 & 3 & -1154.513771 & $\mathrm{~T}$ & cumulative \\
\hline 3.0 & CAS & -1154.513764 & $\mathrm{~T}$ & cumulative \\
\hline 3.5 & 0 & -1154.631333 & $\mathrm{~T}$ & cumulative \\
\hline 3.5 & 1 & -1154.633592 & $\mathrm{~T}$ & cumulative \\
\hline 3.5 & 1 & -1154.633644 & $\mathrm{~T}$ & local \\
\hline 3.5 & 2 & -1154.633661 & $\mathrm{~T}$ & cumulative \\
\hline 3.5 & 3 & -1154.633657 & $\mathrm{~T}$ & cumulative \\
\hline 3.5 & CAS & -1154.633655 & $\mathrm{~T}$ & cumulative \\
\hline 4.5 & 0 & -1154.669490 & $\mathrm{~T}$ & cumulative \\
\hline 4.5 & 1 & -1154.669653 & $\mathrm{~T}$ & cumulative \\
\hline 4.5 & 1 & -1154.669651 & $\mathrm{~T}$ & local \\
\hline 4.5 & 2 & -1154.669655 & $\mathrm{~T}$ & cumulative \\
\hline 4.5 & CAS & -1154.669656 & $\mathrm{~T}$ & cumulative \\
\hline 5.0 & 0 & -1154.671701 & $\mathrm{~T}$ & cumulative \\
\hline 5.0 & 1 & -1154.671740 & $\mathrm{~T}$ & cumulative \\
\hline 5.0 & 1 & -1154.671739 & $\mathrm{~T}$ & local \\
\hline 5.0 & 2 & -1154.671740 & $\mathrm{~T}$ & cumulative \\
\hline 5.0 & CAS & -1154.671740 & $\mathrm{~T}$ & cumulative \\
\hline 6.0 & 0 & -1154.673126 & $\mathrm{~T}$ & cumulative \\
\hline 6.0 & 1 & -1154.673125 & $\mathrm{~T}$ & cumulative \\
\hline 6.0 & 1 & -1154.673130 & $\mathrm{~T}$ & local \\
\hline 6.0 & 2 & -1154.673128 & $\mathrm{~T}$ & cumulative \\
\hline 6.0 & CAS & -1154.673128 & $\mathrm{~T}$ & cumulative \\
\hline 20.0 & 0 & -1154.674761 & $\mathrm{~T}$ & cumulative \\
\hline 20.0 & CAS & -1154.674795 & $\mathrm{~T}$ & cumulative \\
\hline 3.0 & 0 & -1154.483846 & $\mathrm{~F}$ & cumulative \\
\hline 3.0 & 1 & -1154.499365 & $\mathrm{~F}$ & cumulative \\
\hline 3.0 & 2 & -1154.499811 & $\mathrm{~F}$ & cumulative \\
\hline 3.0 & 3 & -1154.499810 & $\mathrm{~F}$ & cumulative \\
\hline 3.0 & CAS & -1154.500000 & $\mathrm{~F}$ & cumulative \\
\hline 3.5 & 0 & -1154.624159 & $\mathrm{~F}$ & cumulative \\
\hline 3.5 & 1 & -1154.628593 & $\mathrm{~F}$ & cumulative \\
\hline 3.5 & 2 & -1154.628614 & $\mathrm{~F}$ & cumulative \\
\hline 3.5 & 3 & -1154.628631 & $\mathrm{~F}$ & cumulative \\
\hline 3.5 & CAS & -1154.628730 & $\mathrm{~F}$ & cumulative \\
\hline 4.5 & 0 & -1154.668048 & $\mathrm{~F}$ & cumulative \\
\hline 4.5 & 1 & -1154.668367 & $\mathrm{~F}$ & cumulative \\
\hline 4.5 & 2 & -1154.668363 & $\mathrm{~F}$ & cumulative \\
\hline 4.5 & CAS & -1154.668432 & $\mathrm{~F}$ & cumulative \\
\hline 5.0 & 0 & -1154.670944 & $\mathrm{~F}$ & cumulative \\
\hline 5.0 & 1 & -1154.671024 & $\mathrm{~F}$ & cumulative \\
\hline 5.0 & 2 & -1154.671024 & $\mathrm{~F}$ & cumulative \\
\hline 5.0 & CAS & -1154.671076 & $\mathrm{~F}$ & cumulative \\
\hline 6.0 & 0 & -1154.672910 & $\mathrm{~F}$ & cumulative \\
\hline 6.0 & 1 & -1154.672918 & $\mathrm{~F}$ & cumulative \\
\hline 6.0 & 2 & -1154.672918 & $\mathrm{~F}$ & cumulative \\
\hline 6.0 & CAS & -1154.672968 & $\mathrm{~F}$ & cumulative \\
\hline 20.0 & 0 & -1154.674755 & $\mathrm{~F}$ & cumulative \\
\hline 20.0 & CAS & -1154.674793 & $\mathrm{~F}$ & cumulative \\
\hline
\end{tabular}


Table 2: Results for the $\mathrm{N}_{4}$ tetrahedron.

\begin{tabular}{|c|c|c|c|c|c|}
\hline \multirow[b]{2}{*}{ Algorithm } & \multicolumn{2}{|c|}{ Defined } & \multirow[b]{2}{*}{$n_{\text {exc }}$} & \multirow[b]{2}{*}{$E / E_{\mathrm{h}}$} & \multirow{2}{*}{$\begin{array}{c}\text { Empirical } \\
S\end{array}$} \\
\hline & $S$ & $S_{z}$ & & & \\
\hline Davidson & 0 & & CAS & -217.691055 & \\
\hline Davidson & 1 & & CAS & $-217.690423^{\mathrm{a}}$ & \\
\hline Davidson & 2 & & CAS & -217.689159 & \\
\hline Davidson & 3 & & CAS & -217.687262 & \\
\hline Davidson & 4 & & CAS & -217.684734 & \\
\hline Davidson & 5 & & CAS & -217.681574 & \\
\hline Davidson & 6 & & CAS & -217.677788 & \\
\hline Davidson & 0 & & 0 & -217.669068 & \\
\hline Davidson & 1 & & 0 & -217.669443 & \\
\hline Davidson & 2 & & 0 & -217.670281 & \\
\hline Davidson & 3 & & 0 & -217.671504 & \\
\hline Davidson & 4 & & 0 & -217.673192 & \\
\hline Davidson & 5 & & 0 & -217.675255 & \\
\hline Davidson & 6 & & 0 & -217.677788 & \\
\hline Davidson & 0 & & 1 & -217.690918 & \\
\hline Davidson & 1 & & 1 & -217.690300 & \\
\hline Davidson & 2 & & 1 & -217.689063 & \\
\hline Davidson & 3 & & 1 & -217.687202 & \\
\hline Davidson & 4 & & 1 & -217.684709 & \\
\hline Davidson & 5 & & 1 & -217.681574 & \\
\hline Davidson & 6 & & 1 & -217.677788 & \\
\hline Davidson & 0 & & 2 & -217.691055 & \\
\hline Davidson & 1 & & 2 & $-217.689963^{b}$ & \\
\hline Davidson & 2 & & 2 & -217.689159 & \\
\hline Davidson & 3 & & 2 & -217.687262 & \\
\hline Davidson & 4 & & 2 & -217.684734 & \\
\hline Davidson & 5 & & 2 & -217.681574 & \\
\hline Davidson & 6 & & 2 & -217.677788 & \\
\hline FCIQMC & & 0 & CAS & -217.690927 & 0.017992 \\
\hline FCIQMC & & 1 & CAS & -217.690294 & 0.999335 \\
\hline FCIQMC & & 2 & CAS & -217.689108 & 2.000011 \\
\hline FCIQMC & & 3 & CAS & -217.687259 & 2.999996 \\
\hline FCIQMC & & 4 & CAS & -217.684734 & 4.000000 \\
\hline FCIQMC & & 5 & CAS & -217.681574 & 5.000000 \\
\hline FCIQMC & & 6 & CAS & -217.677788 & 6.000000 \\
\hline FCIQMC & & 0 & 1 & -217.690805 & 0.014956 \\
\hline FCIQMC & & 1 & 1 & -217.690190 & 0.998939 \\
\hline FCIQMC & & 2 & 1 & -217.689025 & 2.000015 \\
\hline FCIQMC & & 3 & 1 & -217.687200 & 3.000002 \\
\hline FCIQMC & & 4 & 1 & -217.684709 & 4.000000 \\
\hline FCIQMC & & 5 & 1 & -217.681574 & 5.000000 \\
\hline FCIQMC & & 6 & 1 & -217.677788 & 6.000000 \\
\hline FCIQMC & & 0 & 2 & -217.690927 & 0.018142 \\
\hline FCIQMC & & 1 & 2 & -217.690294 & 1.001640 \\
\hline FCIQMC & & 2 & 2 & -217.689111 & 2.000047 \\
\hline FCIQMC & & 3 & 2 & -217.687259 & 2.999990 \\
\hline FCIQMC & & 4 & 2 & -217.684734 & 4.000000 \\
\hline FCIQMC & & 5 & 2 & -217.681574 & 5.000000 \\
\hline FCIQMC & & 6 & 2 & -217.677788 & 6.000000 \\
\hline
\end{tabular}

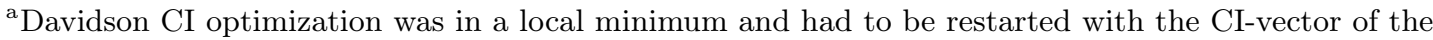
Davidson GAS $n_{\mathrm{exc}}=1$ calculation.

${ }^{\mathrm{b}}$ Davidson CI optimization was in a local minimum. For technical reasons it could not be restarted from converged CI-solutions.
} 
Table 3: Results for the $\mathrm{Fe}_{4} \mathrm{~S}_{4}$ cluster. The spin-pure GUGA results are taken from reference [12].

\begin{tabular}{|c|c|c|c|c|c|}
\hline basis & $S$ & $n_{\text {exc }}$ & $E / E_{\mathrm{h}}$ & $\sigma(E) / \mathrm{m} E_{\mathrm{h}}$ & $(E-E(\mathrm{GUGA} F C \mathrm{~F})) / \mathrm{m} E_{\mathrm{h}}$ \\
\hline slater & 0 & 1 & -8432.54411810 & 0.00080 & 1.05553 \\
\hline slater & 0 & 2 & -8432.54459286 & 0.00071 & 0.58077 \\
\hline slater & 0 & CAS & -8432.54459396 & 0.00079 & 0.57967 \\
\hline slater & 1 & 1 & -8432.54311601 & 0.00755 & 1.51837 \\
\hline slater & 1 & 2 & -8432.54350770 & 0.00791 & 1.12668 \\
\hline slater & 1 & CAS & -8432.54351238 & 0.00577 & 1.12200 \\
\hline slater & 2 & 1 & -8432.54200918 & 0.08352 & 1.57917 \\
\hline slater & 2 & 2 & -8432.54224563 & 0.03242 & 1.34272 \\
\hline slater & 2 & CAS & -8432.54225342 & 0.07292 & 1.33493 \\
\hline slater & 3 & 1 & -8432.54095658 & 0.13840 & 1.11660 \\
\hline slater & 3 & 2 & -8432.54123450 & 0.15041 & 0.83868 \\
\hline slater & 3 & CAS & -8432.54134563 & 0.10314 & 0.72755 \\
\hline slater & 4 & 1 & -8432.53964877 & 0.07697 & 0.52708 \\
\hline slater & 4 & 2 & -8432.53982452 & 0.09787 & 0.35133 \\
\hline slater & 4 & CAS & -8432.53993122 & 0.04333 & 0.24463 \\
\hline slater & 5 & 1 & -8432.53765165 & 0.04632 & 0.30189 \\
\hline slater & 5 & 2 & -8432.53777222 & 0.02443 & 0.18132 \\
\hline slater & 5 & CAS & -8432.53779243 & 0.02172 & 0.16111 \\
\hline slater & 6 & 1 & -8432.53525882 & 0.01031 & 0.21119 \\
\hline slater & 6 & 2 & -8432.53551695 & 0.04561 & -0.04694 \\
\hline slater & 6 & CAS & -8432.53550026 & 0.02918 & -0.03025 \\
\hline slater & 7 & 1 & -8432.53272305 & 0.02581 & 0.06260 \\
\hline slater & 7 & 2 & -8432.53275652 & 0.04259 & 0.02913 \\
\hline slater & 7 & CAS & -8432.53278919 & 0.00508 & -0.00354 \\
\hline slater & 8 & 1 & -8432.52997451 & 0.00143 & 0.02600 \\
\hline slater & 8 & 2 & -8432.52999146 & 0.00161 & 0.00905 \\
\hline slater & 8 & CAS & -8432.52996841 & 0.00248 & 0.03210 \\
\hline slater & 9 & 1 & -8432.52720956 & 0.00044 & 0.00510 \\
\hline slater & 9 & 2 & -8432.52721032 & 0.00032 & 0.00434 \\
\hline slater & 9 & CAS & -8432.52722031 & 0.00031 & -0.00565 \\
\hline slater & 10 & 1 & -8432.52452663 & 0.00000 & 0.00025 \\
\hline slater & 10 & 2 & -8432.52452663 & 0.00000 & 0.00025 \\
\hline slater & 10 & CAS & -8432.52452663 & 0.00000 & 0.00025 \\
\hline GUGA & 0 & CAS & -8432.54517363 & & 0 \\
\hline GUGA & 1 & CAS & -8432.54463438 & & 0 \\
\hline GUGA & 2 & CAS & -8432.54358835 & & 0 \\
\hline GUGA & 3 & CAS & -8432.54207318 & & 0 \\
\hline GUGA & 4 & CAS & -8432.54017585 & & 0 \\
\hline GUGA & 5 & CAS & -8432.53795354 & & 0 \\
\hline GUGA & 6 & CAS & -8432.53547001 & & 0 \\
\hline GUGA & 7 & CAS & -8432.53278565 & & 0 \\
\hline GUGA & 8 & CAS & -8432.53000051 & & 0 \\
\hline GUGA & 9 & CAS & -8432.52721466 & & 0 \\
\hline GUGA & 10 & CAS & -8432.52452688 & & 0 \\
\hline
\end{tabular}




\section{Evaluate $\hat{S}^{2}$ with 2-RDM}

The aim is to bring the operator into the same format as the reduced density matrices. We start with

$$
\hat{S}^{2}=\hat{S}_{+} \hat{S}_{-}+\hat{S}_{z}\left(\hat{S}_{z}-1\right)
$$

and

$$
\begin{aligned}
& \hat{S}_{+}=\sum_{p} a_{p \alpha}^{\dagger} a_{p \beta} \\
& \hat{S}_{-}=\sum_{p} a_{p \beta}^{\dagger} a_{p \alpha} \\
& \hat{S}_{z}=\frac{1}{2} \sum_{p}\left(a_{p \alpha}^{\dagger} a_{p \alpha}-a_{p \beta}^{\dagger} a_{p \beta}\right)
\end{aligned}
$$

We evaluate:

$$
\hat{S}_{+} \hat{S}_{-}=\sum_{p} a_{p \alpha}^{\dagger} a_{p \alpha}-\sum_{p q} a_{p \alpha}^{\dagger} a_{q \beta}^{\dagger} a_{p \beta} a_{q \alpha}
$$

and

$$
\begin{aligned}
4 \hat{S}_{z}\left(\hat{S}_{z}-2\right)= & \sum_{p q}\left(a_{p \alpha}^{\dagger} a_{p \alpha} a_{q \alpha}^{\dagger} a_{q \alpha}-a_{p \alpha}^{\dagger} a_{p \alpha} a_{q \beta}^{\dagger} a_{q \beta}-a_{p \beta}^{\dagger} a_{p \beta} a_{q \alpha}^{\dagger} a_{q \alpha}+a_{p \beta}^{\dagger} a_{p \beta} a_{q \beta}^{\dagger} a_{q \beta}\right) \\
& -2 \sum_{p}\left(a_{p \alpha}^{\dagger} a_{p \alpha}-a_{p \beta}^{\dagger} a_{p \beta}\right) .
\end{aligned}
$$

We note the following identities:

$$
\begin{aligned}
a_{p \alpha}^{\dagger} a_{p \alpha} a_{q \alpha}^{\dagger} a_{q \alpha} & =\delta^{p q} a_{p \alpha}^{\dagger} a_{q \alpha}-a_{p \alpha}^{\dagger} a_{q \alpha}^{\dagger} a_{p \alpha} a_{q \alpha} \\
-a_{p \alpha}^{\dagger} a_{p \alpha} a_{q \beta}^{\dagger} a_{q \beta} & =-\underbrace{\delta^{p \alpha q \beta} a_{p \alpha}^{\dagger} a_{q \beta}}_{0}+a_{p \alpha}^{\dagger} a_{q \beta}^{\dagger} a_{p \alpha} a_{q \beta}=a_{p \alpha}^{\dagger} a_{q \beta}^{\dagger} a_{p \alpha} a_{q \beta} \\
-a_{p \beta}^{\dagger} a_{p \beta} a_{q \alpha}^{\dagger} a_{q \alpha} & =-\underbrace{\delta^{p \beta q \alpha} a_{p \beta}^{\dagger} a_{q \alpha}}_{0}+a_{p \beta}^{\dagger} a_{q \alpha}^{\dagger} a_{p \beta} a_{q \alpha}=a_{p \beta}^{\dagger} a_{q \alpha}^{\dagger} a_{p \beta} a_{q \alpha} \\
a_{p \beta}^{\dagger} a_{p \beta} a_{q \beta}^{\dagger} a_{q \beta} & =\delta^{p q} a_{p \beta}^{\dagger} a_{q \beta}-a_{p \beta}^{\dagger} a_{q \beta}^{\dagger} a_{p \beta} a_{q \beta}
\end{aligned}
$$

Now we can write:

$$
\begin{aligned}
4 \hat{S}_{z}\left(\hat{S}_{z}-2\right)= & -\sum_{p} a_{p \alpha}^{\dagger} a_{p \alpha}+3 \sum_{p} a_{p \beta}^{\dagger} a_{p \beta} \\
& -\sum_{p \neq q}\left(a_{p \alpha}^{\dagger} a_{q \alpha}^{\dagger} a_{p \alpha} a_{q \alpha}+a_{p \beta}^{\dagger} a_{q \beta}^{\dagger} a_{p \beta} a_{q \beta}\right) \\
& +\sum_{p q}\left(a_{p \alpha}^{\dagger} a_{q \beta}^{\dagger} a_{p \alpha} a_{q \beta}+a_{p \beta}^{\dagger} a_{q \alpha}^{\dagger} a_{p \beta} a_{q \alpha}\right) .
\end{aligned}
$$


We conclude:

$$
\begin{aligned}
\hat{S}^{2}= & \hat{S}_{+} \hat{S}_{-}+\hat{S}_{z}\left(\hat{S}_{z}-1\right) \\
= & \frac{3}{4} \sum_{p}\left(a_{p \alpha}^{\dagger} a_{p \alpha}+a_{p \beta}^{\dagger} a_{p \beta}\right) \\
& -\frac{1}{4} \sum_{p \neq q}\left(a_{p \alpha}^{\dagger} a_{q \alpha}^{\dagger} a_{p \alpha} a_{q \alpha}+a_{p \beta}^{\dagger} a_{q \beta}^{\dagger} a_{p \beta} a_{q \beta}\right) \\
& +\frac{1}{4} \sum_{p q}\left(2 a_{p \alpha}^{\dagger} a_{q \beta}^{\dagger} a_{p \alpha} a_{q \beta}-4 a_{p \alpha}^{\dagger} a_{q \beta}^{\dagger} a_{p \beta} a_{q \alpha}\right)
\end{aligned}
$$

\section{References}

(1) Widmark, P.-O.; Malmqvist, P.-A.; Roos, B. O. Theoret. Chim. Acta 1990, 77, 291-306.

(2) Roos, B. O.; Lindh, R.; Malmqvist, P.-Å.; Veryazov, V.; Widmark, P.-O. J. Phys. Chem. A 2004, 108, 2851-2858.

(3) Aquilante, F.; Pedersen, T. B.; Lindh, R. J. Chem. Phys. 2007, 126, 194106.

(4) Aquilante, F.; Lindh, R.; Bondo Pedersen, T. J. Chem. Phys. 2007, $127,114107$.

(5) Aquilante, F.; Pedersen, T. B.; Lindh, R.; Roos, B. O.; Sánchez de Merás, A.; Koch, H. J. Chem. Phys. 2008, 129, 024113.

(6) Aquilante, F.; Gagliardi, L.; Pedersen, T. B.; Lindh, R. J. Chem. Phys. 2009, 130, 154107.

(7) Pedersen, T. B.; Aquilante, F.; Lindh, R. Theor Chem Acc 2009, 124, 1-10.

(8) Weser, O.; Freitag, L.; Guther, K.; Alavi, A.; Li Manni, G. Int. J. Quantum Chem. 2021, 121, 26454-26467.

(9) Li Manni, G.; Alavi, A. J. Phys. Chem. A 2018, 122, 4935-4947.

(10) Guther, K. et al. J. Chem. Phys. 2020, 153, 34107-34131.

(11) Fdez. Galván, I. et al. J. Chem. Theory Comput. 2019, 15, 5925-5964.

(12) Dobrautz, W.; Weser, O.; Bogdanov, N.; Alavi, A.; Manni, G. L. arXiv 2021, 2106.07775. 